CRYSTALLOGRAPHIC COMMUNICATIONS

ISSN 2056-9890

Received 11 November 2020

Accepted 22 December 2020

Edited by A. S. Batsanov, University of Durham, England

Keywords: crystal structure; natural product; lawinal; flavanone.

CCDC reference: 2051848

Supporting information: this article has supporting information at journals.iucr.org/e

\section{Isolation and crystal structure of lawinal}

\author{
Virayu Suthiphasilp, ${ }^{\text {a }}$ Pornphimol Meesakul, ${ }^{a}$ Christopher Richardson, ${ }^{b}$ Stephen G. \\ Pyne $^{\mathrm{b}}$ and Surat Laphookhieo ${ }^{\mathrm{a} *}$
}

${ }^{a}$ Center of Chemical Innovation for Sustainability (CIS) and School of Science, Mae Fah Luang University, Chiang Rai 57100, Thailand, and ${ }^{\mathbf{b}}$ School of Chemistry and Molecular Bioscience, University of Wollongong, Wollongong, New South Wales 2522, Australia. *Correspondence e-mail: surat.lap@mfu.ac.th

The structure of the natural product lawinal [systematic name: (-)-(2S)-5,7dihydroxy-6-methyl-4-oxo-2-phenylchromane-8-carbaldehyde, $\left.\mathrm{C}_{17} \mathrm{H}_{14} \mathrm{O}_{5}\right]$ at $150 \mathrm{~K}$ is reported. The compound crystallizes with monoclinic (I2) symmetry and with $Z^{\prime}=2$. The absolute configuration could not be determined reliably from $\mathrm{X}$-ray analysis only. However, our analysis returns the $S$-configuration at the $\mathrm{C}-2$ position, consistent with previous stereochemical assignment from specific rotation. The independent molecules form into alternating hydrogenbonded chains with $\mathrm{C}-\mathrm{H} \cdots \mathrm{O}=\mathrm{CH}$ intermolecular linkages that run parallel to the crystallographic $a$ axis and are extended into the $a c$ plane by $\pi-\pi$ interactions between their phenyl substituents.

\section{Chemical context}

The small flowering plants of the Desmos genus belong to the Annonaceae family, which comprises about 33 species and is distributed widely throughout Southern Asia and northern Australia (Brophy et al., 2002; Clement et al., 2017). Several species of this genus have been used as Chinese folk medicines (Wu et al., 2003). The aerial part of $D$. chinensis has been used as an analgesic agent, and to treat vertigo, and parturition (Kummee \& Intaraksa, 2008; Rahman et al., 2003). In Thailand it is widely used traditionally to treat fever and dysentery (Bunyapraphatsara et al., 2000). The petroleum ether extracts of $D$. cochinchinensis roots have mainly been explored for their antimalarial activity (Liao et al., 1989). The Desmos genus is well known as an abundant source of flavonoids (Meesakul et al., 2019; Bajgai et al., 2011; Kuo et al., 2015), and their $2 S$ absolute configuration has been commonly found (Meesakul et al., 2019; Kuo et al., 2015). Flavonoids exhibit interesting biological activities, including inhibition of HIV-1 replication in $\mathrm{H} 9$ lymphocytic cells (Wu et al., 2003), antibacterial properties (Liao et al., 1989) and show activities as $\alpha$ glucosidase inhibitors (Meesakul et al., 2019), antioxidants (Miller, 1996), aromatase and lipoxygenase inhibitors (Bajgai et al., 2011).

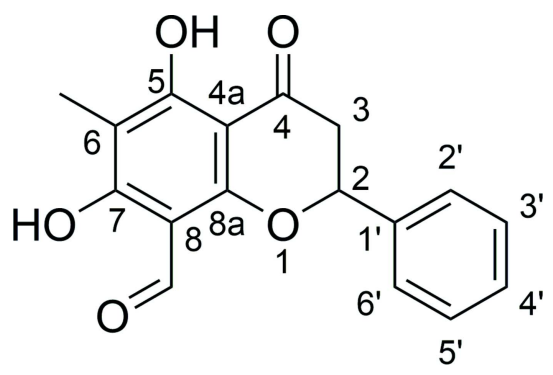


Table 1

Hydrogen-bond geometry $\left(\AA{ }^{\circ}\right)$.

\begin{tabular}{lllll}
\hline$D-\mathrm{H} \cdots A$ & $D-\mathrm{H}$ & $\mathrm{H} \cdots A$ & $D \cdots A$ & $D-\mathrm{H} \cdots A$ \\
\hline $\mathrm{C} 3-\mathrm{H} 3 B \cdots \mathrm{O} 9 A^{\mathrm{i}}$ & 0.99 & 2.32 & $3.252(3)$ & 158 \\
$\mathrm{C} 3 A-\mathrm{H} 3 A B \cdots \mathrm{O} 99^{\mathrm{ii}}$ & 0.99 & 2.31 & $3.248(3)$ & 157 \\
$\mathrm{O} 7 A-\mathrm{H} 7 A \cdots \mathrm{O} 9 A$ & $0.94(3)$ & $1.67(3)$ & $2.572(2)$ & $159(4)$ \\
$\mathrm{O} 5-\mathrm{H} 5 \cdots \mathrm{O} 4$ & $0.94(3)$ & $1.70(3)$ & $2.579(2)$ & $154(3)$ \\
$\mathrm{O} 7-\mathrm{H} 7 \cdots \mathrm{O} 9$ & $0.95(3)$ & $1.67(3)$ & $2.585(2)$ & $162(3)$ \\
O5A-H5A $\cdots \mathrm{O} 4 A$ & $0.94(3)$ & $1.70(3)$ & $2.574(2)$ & $153(3)$ \\
\hline
\end{tabular}

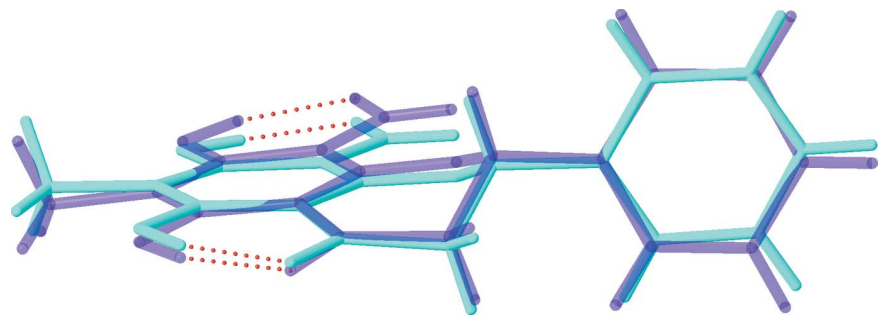

Symmetry codes: (i) $-x+\frac{3}{2}, y-\frac{1}{2},-z+\frac{1}{2}$; (ii) $-x+\frac{1}{2}, y+\frac{1}{2},-z+\frac{1}{2}$.

Herein, we report the isolation and crystal structure of the flavonoid, (-)(2S)-5,7-dihydroxy-6-methyl-4-oxo-2-phenylchromane-8-carbaldehyde, commonly known as lawinal, isolated from the twig extract of $D$. dumosus.

\section{Structural commentary}

Lawinal crystallizes in the space group $I 2$ with $Z^{\prime}=2$. Because of the large standard deviation of the Flack parameter [ -0.1 (5)], the absolute configuration cannot be assigned from the X-ray data (Parsons et al., 2013). We explored applying the Bayesian statistical approach promoted by Hooft et al. (2008). Given that the compound comes from a natural product source and thus should be enantiopure, the analysis, as implemented in PLATON (Spek, 2020), returned a P2(true) value of 0.992 for the $S$-configuration at C2 in each molecule (Fig. 1). This is consistent with the stereochemical assignment by the method of specific rotation (Prawat et al., 2012; Wu et al., 2005).

The unique molecules adopt extremely similar conformations and an overlay of the molecular structures is shown in Fig. 2. The hydroxyl groups attached to $\mathrm{C} 5$ and $\mathrm{C} 7$ on each unique molecule act as hydrogen-bond donors to the ketone and aldehyde functionalities, respectively. The positions of the hydroxyl hydrogen atoms were refined, the relatively long $D-$ $\mathrm{H}$ distances (Table 1) indicating strong intramolecular stabilization. The hydrogen bond $\mathrm{O} 7-\mathrm{H} 7 \cdots \mathrm{O} 9$ is responsible for

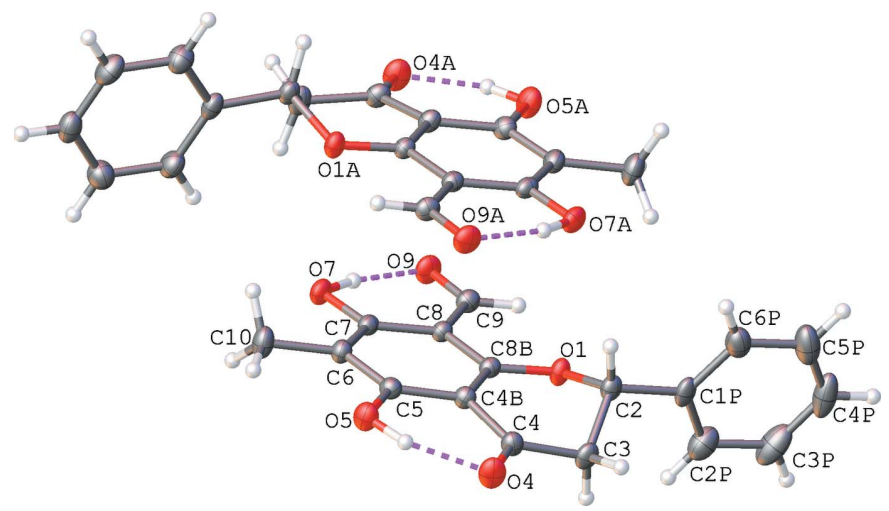

Figure 1

The contents of the asymmetric unit with complete atom labelling of one molecule and selected heteroatom labelling of the second molecule, for clarity. Intramolecular hydrogen bonds are shown as dashed magenta lines. Displacement ellipsoids are plotted at the $50 \%$ probability level.

Figure 2

An overlay of the independent molecules in the asymmetric unit. The dotted lines represent the intramolecular hydrogen bonds.

bringing the aldehyde group into approximate coplanarity with the chromanone ring system. In contrast, the phenyl substituents attached to $\mathrm{C} 2$ in each molecule are approximately orthogonal to the chromanone ring systems [plane-toplane angles of 99.4 (1) and $97.5(1)^{\circ}$ to the phenyl rings of the chromanones].

\section{Supramolecular features}

The shortest intermolecular contacts to hydrogen-bond acceptors of the unique molecules come from the pseudoequatorial $\mathrm{C}-\mathrm{H}$ bonds in the $\mathrm{CH}_{2}$ moieties of the chromanone rings to the aldehyde oxygen atoms, $\mathrm{O} 9$ and $\mathrm{O} 9 \mathrm{~A}$ (Table 1). These $\mathrm{C}-\mathrm{H} \cdots \mathrm{O}=\mathrm{CH}$ connections assemble the unique molecules into alternating chains that propagate parallel to the crystallographic $a$-axis, as shown in Fig. 3. The supramolecular alignment of these hydrogen bonded chains are controlled by $\pi-\pi$ interactions of phenyl rings from

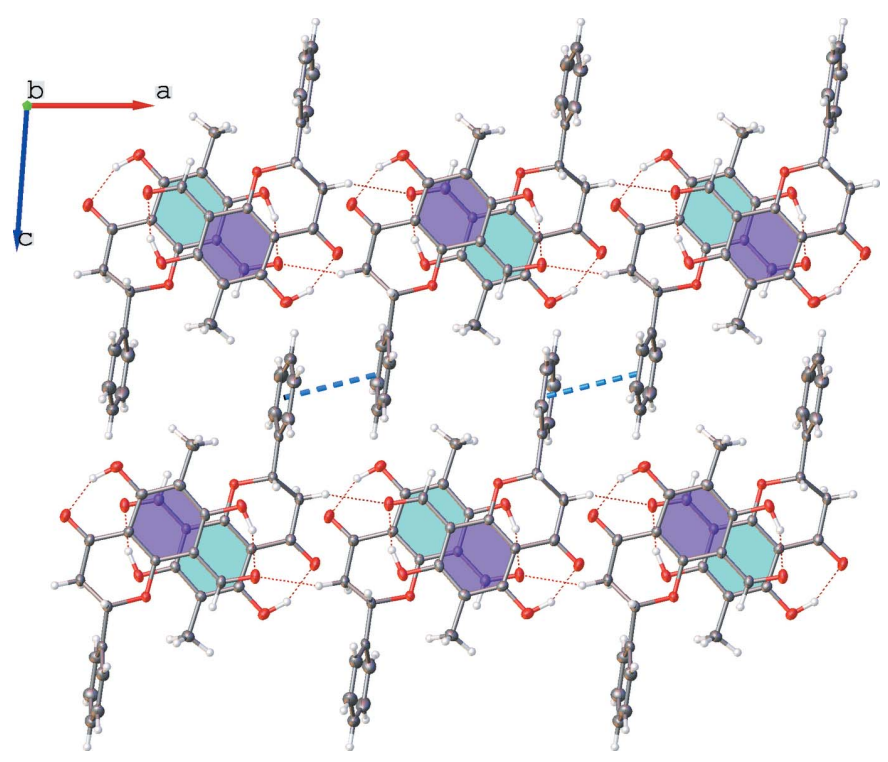

Figure 3

A view parallel to the crystallographic $b$-axis, with intramolecular and intermolecular hydrogen bonds shown as dotted red lines and the $\pi-\pi$ interactions as dashed blue lines. The intermolecular hydrogen bonds link molecules into chains propagating along the crystallographic $a$-axis direction and the $\pi-\pi$ interactions link the hydrogen-bonded chains into two-dimensional sheets in the crystallographic ac plane. Displacement ellipsoids are plotted at the $50 \%$ probability level. 
adjacent chains. This links the chains into two-dimensional sheets in the ac plane. The plane-to-plane angle between phenyl rings is $4.7(1)^{\circ}$ and the distance from plane centroid to plane centroid, as indicated by the blue dashed line in Fig. 3, is 3.821 (2) $\AA$.

\section{Synthesis and crystallization}

\section{Plant Material}

Desmos dumosus twigs were collected from Doi Tung, Chiang Rai Province, Thailand, in February 2016. The plant was identified by Mr Matin Van de Bult (Doi Tung Development Project, Chiang Rai, Thailand). The specimen (MFUNPR0110) was deposited at Mae Fah Luang University's Natural Products Research Laboratory.

\section{Extraction and Isolation}

Air-dried twigs of $D$. dumosus $(7.00 \mathrm{~kg})$ were extracted for three days at room temperature with EtOAc $(20 \mathrm{~L})$. Removal of the solvent under reduced pressure provided the crude extract $(92.7 \mathrm{~g})$, which was subjected to column chromatography over silica gel using a gradient of hexanes and EtOAc (100\% hexanes to $100 \%$ EtOAc) to afford 12 fractions (D1D12). Fraction D5 (7.70 g) was further fractionated by column chromatography over Sephadex-LH 20 resin eluting with $100 \% \mathrm{MeOH}$ to provide nine subfractions (D5A-D5I). Subfraction D5E (1.45 g) was further separated by column chromatography over silica gel $(1: 4, v / v$ EtOAc/hexanes) to give lawinal $(35.5 \mathrm{mg})$ as a faint yellow-coloured solid.

\section{Crystallization and characterization data}

Crystals grew from slow evaporation of a 1:4 dichloromethane:methanol solution. M.p. 488-489 K [Lit. (Prawat et al., 2012) $487 \mathrm{~K}] ;[\alpha]_{\mathrm{D}}{ }^{25}-52.4\left(\right.$ c $\left.0.2, \mathrm{CH}_{2} \mathrm{Cl}_{2}\right) ; \mathrm{ECD}(3.4 \times$ $\left.10^{-4}\right) \lambda_{\max }(\Delta \varepsilon) 298(+4.66), 276(-4.88)$, and $228(+3.82) ;{ }^{1} \mathrm{H}$ $\operatorname{NMR}\left(\mathrm{CDCl}_{3}, 500 \mathrm{MHz}\right) \delta_{\mathrm{H}} 12.85(1 \mathrm{H}, s, \mathrm{OH}-5), 13.00(1 \mathrm{H}, s$, $\mathrm{OH}-7), 10.11(1 \mathrm{H}, s, \mathrm{CHO}), 7.45\left(5 \mathrm{H}, m, \mathrm{H}-2^{\prime}-\mathrm{H}-6^{\prime}\right), 5.57(1 \mathrm{H}$, $d d, J=13.0,3.2 \mathrm{~Hz}, \mathrm{H}-2), 3.16(1 \mathrm{H}, d d, J=17.3,13.0 \mathrm{~Hz}$, $\mathrm{H} \alpha-3), 2.93(1 \mathrm{H}, d d, J=17.3,3.2 \mathrm{~Hz}, \mathrm{H} \beta-3), 2.02\left(3 \mathrm{H}, s, \mathrm{CH}_{3}\right)$; ${ }^{13} \mathrm{C}$ NMR $\left(\mathrm{CDCl}_{3}, 125 \mathrm{MHz}\right) \delta_{\mathrm{C}} 6.0\left(\mathrm{CH}_{3}\right), 42.8(\mathrm{C}-3), 80.3$ (C2), 101.3 (C4a), 104.1 (C8), 105.7 (C6), $126.1\left(\mathrm{C}^{\prime}, \mathrm{C6}^{\prime}\right)$, 129.1 (C3', C4', C5'), 137.6 (C1'), 164.7 (C8a), 166.6 (C5), 168.8 (C7), 191.3 (CHO), 195.3 (C4).

\section{Refinement}

The data were collected using Mo $K \alpha$ radiation, therefore anomalous dispersion effects are small. The crystal structure itself is pseudo-centrosymmetric. Indeed, a structural solution can be successfully obtained in a centrosymmetric space group, although this results in an unsatisfactory refinement, with apparent disorder about the stereogenic center, as expected. The actual inversion symmetry is, of course, incompatible with the natural origin and optical activity of the compound. Crystal data, data collection and structure refinement details are summarized in Table 2. Tertiary $\mathrm{C}(\mathrm{H})$, secondary $\mathrm{C}(\mathrm{H}, \mathrm{H})$, primary $\mathrm{C}(\mathrm{H}, \mathrm{H}, \mathrm{H})$ and aromatic $\mathrm{H}$ atoms were placed in geometrically idealized positions $(\mathrm{C}-\mathrm{H}=1.00$, $0.99,0.98$, and $0.95 \AA$, respectively) and refined in riding
Table 2

Experimental details.

\begin{tabular}{|c|c|}
\hline \multicolumn{2}{|l|}{ Crystal data } \\
\hline Chemical formula & $\mathrm{C}_{17} \mathrm{H}_{14} \mathrm{O}_{5}$ \\
\hline$M_{\mathrm{r}}$ & 298.28 \\
\hline Crystal system, space group & Monoclinic, $I 2$ \\
\hline Temperature $(\mathrm{K})$ & 150 \\
\hline$a, b, c(\AA)$ & $\begin{array}{l}18.9581(14), 6.6461(4), \\
\quad 22.4043(16)\end{array}$ \\
\hline$\beta\left({ }^{\circ}\right)$ & $94.163(7)$ \\
\hline$V\left(\AA^{3}\right)$ & $2815.4(3)$ \\
\hline$Z$ & 8 \\
\hline Radiation type & Мо $K \alpha$ \\
\hline$\mu\left(\mathrm{mm}^{-1}\right)$ & 0.10 \\
\hline Crystal size $(\mathrm{mm})$ & $0.42 \times 0.19 \times 0.16$ \\
\hline \multicolumn{2}{|l|}{ Data collection } \\
\hline Diffractometer & Rigaku XtaLAB Mini II \\
\hline Absorption correction & $\begin{array}{l}\text { Multi-scan (CrysAlis PRO; Rigaku } \\
\text { OD, 2018) }\end{array}$ \\
\hline$T_{\min }, T_{\max }$ & $0.728,1.000$ \\
\hline $\begin{array}{l}\text { No. of measured, independent and } \\
\text { observed }[I>2 \sigma(I)] \text { reflections }\end{array}$ & $33601,7914,6451$ \\
\hline$R_{\text {int }}$ & 0.072 \\
\hline$(\sin \theta / \lambda)_{\max }\left(\AA^{-1}\right)$ & 0.718 \\
\hline \multicolumn{2}{|l|}{ Refinement } \\
\hline$R\left[F^{2}>2 \sigma\left(F^{2}\right)\right], w R\left(F^{2}\right), S$ & $0.053,0.144,1.02$ \\
\hline No. of reflections & 7914 \\
\hline No. of parameters & 415 \\
\hline No. of restraints & 1 \\
\hline $\mathrm{H}$-atom treatment & $\begin{array}{l}\mathrm{H} \text { atoms treated by a mixture of } \\
\text { independent and constrained } \\
\text { refinement }\end{array}$ \\
\hline$\Delta \rho_{\max }, \Delta \rho_{\min }\left(\mathrm{e} \AA^{-3}\right)$ & $0.39,-0.32$ \\
\hline Absolute structure & $\begin{array}{l}\text { Flack } x \text { determined using } 2350 \\
\quad \text { quotients }\left[\left(I^{+}\right)-\left(I^{-}\right)\right] /\left[\left(I^{+}\right)+\left(I^{-}\right)\right] \\
\quad \text { (Parsons } \text { et al. } 2013)\end{array}$ \\
\hline Absolute structure parameter & $-0.1(5)$ \\
\hline
\end{tabular}

Computer programs: CrysAlis PRO (Rigaku OD, 2018), SHELXS (Sheldrick, 2008), SHELXL (Sheldrick, 2015) and OLEX2 (Dolomanov et al., 2009).

models with $U_{\text {iso }}(\mathrm{H})=1.2 U_{\text {eq }}(\mathrm{C})$ or $1.5 U_{\text {eq }}(\mathrm{C})$. The methyl group attached to $\mathrm{C}-6$ was refined as a rotating body. The hydroxylic $\mathrm{H}$ atoms were refined unconstrained in isotropic approximation.

\section{Acknowledgements}

The University of Wollongong is acknowledged for providing laboratory facilities.

\section{Funding information}

Funding for this research was provided by: Thailand Science Research and Innovation (grant No. DBG6280007; studentship No. PHD/0133/2559 to V. Suthiphasilp); Mae Fah Luang University.

\section{References}

Bajgai, S. P., Prachyawarakorn, V., Mahidol, C., Ruchirawat, S. \& Kittakoop, P. (2011). Phytochemistry, 72, 2062-2067.

Brophy, J. J., Goldsack, R. J. \& Forster, P. I. (2002). J. Essent. Oil Res. 14, 298-301.

Bunyapraphatsara, N., Dechsree, S., Yoosook, C., Herunsalee, A. \& Panpisutchai, Y. (2000). Phytomedicine, 6, 421-424. 
Clement, J. A., Ondeyka, J. G. \& Goetz, M. A. (2017). Phytochemistry Lett. 22, 117-121.

Dolomanov, O. V., Bourhis, L. J., Gildea, R. J., Howard, J. A. K. \& Puschmann, H. (2009). J. Appl. Cryst. 42, 339-341.

Hooft, R. W. W., Straver, L. H. \& Spek, A. L. (2008). J. Appl. Cryst. 41, 96-103.

Kummee, S. \& Intaraksa, N. (2008). Songklanakarin J. Sci. Technol. 30, 635-639.

Kuo, P. C., Thang, T. D., Huang, G. J., Huang, B. S., Hoa, L. T. M., Yang, M. \& Wu, T. (2015). Chem. Nat. Compd. 51, 152-155.

Liao, S. X., Han, G. Y., Zhang, Y. R., Zheng, Q. T. \& He, C. H. (1989). Yaо хие хие bao. 24, 110-113.

Meesakul, P., Richardson, C., Pyne, S. G. \& Laphookhieo, S. (2019). J. Nat. Prod. 82, 741-747.

Miller, A. L. (1996). Alt. Med. Rev. 1, 103-111.
Parsons, S., Flack, H. D. \& Wagner, T. (2013). Acta Cryst. B69, 249259.

Prawat, U., Phupornprasert, D., Butsuri, A., Salae, A.-W., Boonsri, S. \& Tuntiwachwuttikul, P. (2012). Phytochemistry Lett. 5, 809-813.

Rahman, M. M., Qais, N. \& Rashid, M. A. (2003). Fitoterapia, 74, 511514.

Rigaku OD (2018). CrysAlis PRO. Rigaku Oxford Diffraction, Yarnton, England.

Sheldrick, G. M. (2008). Acta Cryst. A64, 112-122.

Sheldrick, G. M. (2015). Acta Cryst. C71, 3-8.

Spek, A. L. (2020). Acta Cryst. E76, 1-11.

Wu, J. H., Shi, N., Pan, M. X., Wang, X. H. \& Yi, Y. H. (2005). Chin. Pharm. J. 40, 495-497.

Wu, J. H., Wang, X. H., Yi, Y. H. \& Lee, K. H. (2003). Bioorg. Med. Chem. Lett. 13, 1813-1815. 


\section{supporting information}

Acta Cryst. (2021). E77, 75-78 [https://doi.org/10.1107/S2056989020016540]

\section{Isolation and crystal structure of lawinal}

\section{Virayu Suthiphasilp, Pornphimol Meesakul, Christopher Richardson, Stephen G. Pyne and} Surat Laphookhieo

\section{Computing details}

Data collection: CrysAlis PRO (Rigaku OD, 2018); cell refinement: CrysAlis PRO (Rigaku OD, 2018); data reduction: CrysAlis PRO (Rigaku OD, 2018); program(s) used to solve structure: SHELXS (Sheldrick, 2008); program(s) used to refine structure: SHELXL (Sheldrick, 2015); molecular graphics: OLEX2 (Dolomanov et al., 2009); software used to prepare material for publication: OLEX2 (Dolomanov et al., 2009).

(2S)-5,7-Dihydroxy-6-methyl-4-oxo-2-phenylchromane-8-carbaldehyde

Crystal data

$\mathrm{C}_{17} \mathrm{H}_{14} \mathrm{O}_{5}$

$M_{r}=298.28$

Monoclinic, $I 2$

$a=18.9581(14) \AA$

$b=6.6461(4) \AA$

$c=22.4043(16) \AA$

$\beta=94.163(7)^{\circ}$

$V=2815.4(3) \AA^{3}$

$Z=8$

Data collection

Rigaku XtaLAB Mini II diffractometer

Radiation source: fine-focus sealed X-ray tube, Rigaku (Mo) X-ray Source

Graphite monochromator $\omega$ scans

Absorption correction: multi-scan (CrysAlisPro; Rigaku OD, 2018)

$T_{\min }=0.728, T_{\max }=1.000$

Refinement

Refinement on $F^{2}$

Least-squares matrix: full

$R\left[F^{2}>2 \sigma\left(F^{2}\right)\right]=0.053$

$w R\left(F^{2}\right)=0.144$

$S=1.02$

7914 reflections

415 parameters

1 restraint
$F(000)=1248$

$D_{\mathrm{x}}=1.407 \mathrm{Mg} \mathrm{m}^{-3}$

Mo $K \alpha$ radiation, $\lambda=0.71073 \AA$

Cell parameters from 12756 reflections

$\theta=2.1-30.6^{\circ}$

$\mu=0.10 \mathrm{~mm}^{-1}$

$T=150 \mathrm{~K}$

Block, clear colourless

$0.42 \times 0.19 \times 0.16 \mathrm{~mm}$

33601 measured reflections

7914 independent reflections

6451 reflections with $I>2 \sigma(I)$

$R_{\text {int }}=0.072$

$\theta_{\max }=30.7^{\circ}, \theta_{\min }=2.2^{\circ}$

$h=-26 \rightarrow 26$

$k=-9 \rightarrow 9$

$l=-32 \rightarrow 32$

Primary atom site location: structure-invariant direct methods

Hydrogen site location: mixed

$\mathrm{H}$ atoms treated by a mixture of independent and constrained refinement

$w=1 /\left[\sigma^{2}\left(F_{\mathrm{o}}^{2}\right)+(0.0925 P)^{2}\right]$ where $P=\left(F_{\mathrm{o}}^{2}+2 F_{\mathrm{c}}^{2}\right) / 3$

$(\Delta / \sigma)_{\max }<0.001$

$\Delta \rho_{\max }=0.39 \mathrm{e}^{-3}$ 
$\Delta \rho_{\min }=-0.32$ e $\AA^{-3}$
Absolute structure: Flack $x$ determined using 2350 quotients $\left[\left(I^{+}\right)-\left(I^{\prime}\right)\right] /\left[\left(I^{+}\right)+\left(I^{-}\right)\right]$(Parsons $e t$ al., 2013)

Absolute structure parameter: $-0.1(5)$

Special details

Geometry. All esds (except the esd in the dihedral angle between two 1.s. planes) are estimated using the full covariance matrix. The cell esds are taken into account individually in the estimation of esds in distances, angles and torsion angles; correlations between esds in cell parameters are only used when they are defined by crystal symmetry. An approximate (isotropic) treatment of cell esds is used for estimating esds involving l.s. planes.

Fractional atomic coordinates and isotropic or equivalent isotropic displacement parameters $\left(\AA^{2}\right)$

\begin{tabular}{|c|c|c|c|c|}
\hline & $x$ & $y$ & $z$ & $U_{\text {iso }} * / U_{\text {eq }}$ \\
\hline $\mathrm{O} 1$ & $0.57571(8)$ & $0.2436(3)$ & $0.16037(6)$ & $0.0205(4)$ \\
\hline $\mathrm{O} 4$ & $0.73983(8)$ & $0.1600(3)$ & $0.28806(7)$ & $0.0284(4)$ \\
\hline O5 & $0.64990(9)$ & 0.1903 (3) & $0.36865(6)$ & $0.0248(4)$ \\
\hline O7 & $0.40722(8)$ & 0.2205 & $0.30729(7)$ & $0.0220(4)$ \\
\hline O9 & $0.37112(8)$ & 0.2498 & $0.19412(7)$ & $0.0262(4)$ \\
\hline $\mathrm{C} 2$ & $0.64822(11)$ & $0.3054(4)$ & $0.14926(8)$ & $0.0204(4)$ \\
\hline $\mathrm{H} 2$ & 0.655815 & 0.445414 & 0.164727 & $0.024 *$ \\
\hline $\mathrm{C} 3$ & $0.70093(11)$ & $0.1669(4)$ & $0.18367(9)$ & $0.0225(5)$ \\
\hline $\mathrm{H} 3 \mathrm{~A}$ & 0.693728 & 0.026481 & 0.169839 & $0.027 *$ \\
\hline H3B & 0.749831 & 0.207369 & 0.176389 & $0.027^{*}$ \\
\hline $\mathrm{C} 4$ & $0.69015(11)$ & $0.1811(4)$ & $0.24939(9)$ & 0.0205 (4) \\
\hline $\mathrm{C} 5$ & $0.59854(11)$ & $0.2066(3)$ & $0.32465(9)$ & $0.0184(4)$ \\
\hline C6 & $0.52807(11)$ & $0.2131(4)$ & $0.34014(9)$ & $0.0193(5)$ \\
\hline $\mathrm{C} 7$ & $0.47547(11)$ & 0.2219 & $0.29309(9)$ & $0.0173(4)$ \\
\hline $\mathrm{C} 8$ & $0.49093(11)$ & $0.2327(3)$ & $0.23198(9)$ & $0.0163(4)$ \\
\hline C9 & $0.43514(11)$ & $0.2491(4)$ & $0.18501(9)$ & $0.0203(5)$ \\
\hline H9 & 0.447893 & 0.259941 & 0.144908 & $0.024 *$ \\
\hline $\mathrm{C} 10$ & $0.50961(12)$ & $0.2053(5)$ & $0.40436(9)$ & $0.0280(6)$ \\
\hline H10A & 0.488694 & 0.333896 & 0.415220 & $0.042 *$ \\
\hline H10B & 0.552530 & 0.180389 & 0.430374 & $0.042 *$ \\
\hline $\mathrm{H} 10 \mathrm{C}$ & 0.475581 & 0.096640 & 0.409285 & $0.042 *$ \\
\hline $\mathrm{C} 4 \mathrm{~B}$ & $0.61711(12)$ & $0.2130(4)$ & $0.26415(9)$ & $0.0177(4)$ \\
\hline $\mathrm{C} 8 \mathrm{~B}$ & $0.56271(11)$ & $0.2306(3)$ & $0.21864(9)$ & $0.0165(4)$ \\
\hline C1P & $0.65328(11)$ & $0.3085(4)$ & $0.08262(9)$ & $0.0243(5)$ \\
\hline $\mathrm{C} 2 \mathrm{P}$ & $0.64364(13)$ & $0.1363(5)$ & $0.04797(11)$ & $0.0366(6)$ \\
\hline $\mathrm{H} 2 \mathrm{P}$ & 0.633195 & 0.011407 & 0.065971 & $0.044 *$ \\
\hline $\mathrm{C} 3 \mathrm{P}$ & $0.64952(15)$ & $0.1489(6)$ & $-0.01417(12)$ & $0.0462(8)$ \\
\hline H3P & 0.642758 & 0.032115 & -0.038366 & $0.055^{*}$ \\
\hline $\mathrm{C} 4 \mathrm{P}$ & $0.66512(13)$ & $0.3311(7)$ & $-0.04014(10)$ & $0.0448(8)$ \\
\hline $\mathrm{H} 4 \mathrm{P}$ & 0.669125 & 0.338660 & -0.082103 & $0.054^{*}$ \\
\hline $\mathrm{C} 5 \mathrm{P}$ & $0.67485(13)$ & $0.5008(6)$ & $-0.00559(10)$ & $0.0430(7)$ \\
\hline H5P & 0.685672 & 0.625104 & -0.023760 & $0.052 *$ \\
\hline C6P & $0.66898(12)$ & $0.4918(5)$ & $0.05540(10)$ & $0.0324(6)$ \\
\hline H6P & 0.675579 & 0.609940 & 0.079017 & $0.039 *$ \\
\hline O1A & $0.42391(8)$ & $0.7039(3)$ & $0.34098(6)$ & $0.0201(3)$ \\
\hline
\end{tabular}




$\begin{array}{lllll}\text { O4A } & 0.26002(9) & 0.6903(3) & 0.21057(7) & 0.0290(4) \\ \text { O5A } & 0.35043(9) & 0.7514(3) & 0.13220(7) & 0.0280(4) \\ \text { O7A } & 0.59290(8) & 0.7443(3) & 0.19545(7) & 0.0233(4) \\ \text { O9A } & 0.62819(8) & 0.7220(3) & 0.30829(7) & 0.0267(4) \\ \text { C2A } & 0.35218(11) & 0.7575(4) & 0.35631(9) & 0.0206(5) \\ \text { H2A } & 0.345949 & 0.906404 & 0.352371 & 0.025^{*} \\ \text { C3A } & 0.29726(11) & 0.6534(4) & 0.31422(9) & 0.0243(5) \\ \text { H3AA } & 0.299403 & 0.506440 & 0.321265 & 0.029^{*} \\ \text { H3AB } & 0.249480 & 0.700565 & 0.322737 & 0.029^{*} \\ \text { C4A } & 0.30940(12) & 0.6959(4) & 0.25019(9) & 0.0211(4) \\ \text { C5A } & 0.40185(12) & 0.7439(3) & 0.17646(9) & 0.0202(5) \\ \text { C6A } & 0.47233(12) & 0.7487(4) & 0.16168(9) & 0.0212(5) \\ \text { C7A } & 0.52461(11) & 0.7395(3) & 0.20899(9) & 0.0188(4) \\ \text { C8A } & 0.50869(11) & 0.7268(3) & 0.26992(9) & 0.0173(4) \\ \text { C9A } & 0.56451(11) & 0.7198(4) & 0.31718(9) & 0.0204(5) \\ \text { H9A } & 0.551698 & 0.713108 & 0.357350 & 0.024^{*} \\ \text { C10A } & 0.49126(13) & 0.7599(5) & 0.09739(9) & 0.0307(6) \\ \text { H10D } & 0.451210 & 0.712862 & 0.070936 & 0.046^{*} \\ \text { H10E } & 0.532553 & 0.674776 & 0.092114 & 0.046^{*} \\ \text { H10F } & 0.502307 & 0.899468 & 0.087429 & 0.046^{*} \\ \text { C4AA } & 0.38250(12) & 0.7287(4) & 0.23673(9) & 0.0187(4) \\ \text { C8AA } & 0.43682(11) & 0.7208(3) & 0.28291(9) & 0.0167(4) \\ \text { C1PA } & 0.34755(12) & 0.6990(4) & 0.42113(9) & 0.0223(5) \\ \text { C2PA } & 0.33826(12) & 0.5001(4) & 0.43833(9) & 0.0278(5) \\ \text { H2PA } & 0.335466 & 0.396820 & 0.408958 & 0.033^{*} \\ \text { C3PA } & 0.33302(13) & 0.4515(4) & 0.49835(10) & 0.0316(6) \\ \text { H3PA } & 0.326462 & 0.315656 & 0.509901 & 0.038^{*} \\ \text { C4PA } & 0.33746(12) & 0.6031(5) & 0.54122(10) & 0.0302(6) \\ \text { H4PA } & 0.333388 & 0.570378 & 0.582103 & 0.036^{*} \\ \text { C5PA } & 0.34774(13) & 0.8014(5) & 0.52490(10) & 0.0317(6) \\ \text { H5PA } & 0.351439 & 0.904073 & 0.554461 & 0.038^{*} \\ \text { C6PA } & 0.35263(12) & 0.8490(4) & 0.46478(9) & 0.0281(5) \\ \text { H6PA } & 0.359485 & 0.984872 & 0.453403 & 0.034^{*} \\ \text { H7A } & 0.616(2) & 0.742(6) & 0.2338(15) & 0.060(11)^{*} \\ \text { H5 } & 0.6917(17) & 0.176(6) & 0.3492(13) & 0.049(9)^{*} \\ \text { H7 } & 0.3847(18) & 0.226(6) & 0.2681(13) & 0.042(9)^{*} \\ \text { H5A } & 0.3078(17) & 0.733(5) & 0.1507(13) & 0.041(9)^{*} \\ & & & & \end{array}$

Atomic displacement parameters $\left(\AA^{2}\right)$

\begin{tabular}{lllllll}
\hline & $U^{11}$ & $U^{22}$ & $U^{33}$ & $U^{12}$ & $U^{13}$ & $U^{23}$ \\
\hline O1 & $0.0133(7)$ & $0.0324(10)$ & $0.0158(6)$ & $-0.0003(6)$ & $0.0016(5)$ & $-0.0005(6)$ \\
O4 & $0.0144(7)$ & $0.0437(11)$ & $0.0265(8)$ & $0.0028(7)$ & $-0.0028(6)$ & $0.0037(7)$ \\
O5 & $0.0194(8)$ & $0.0352(10)$ & $0.0193(7)$ & $0.0010(7)$ & $-0.0026(6)$ & $0.0004(7)$ \\
O7 & $0.0152(8)$ & $0.0282(10)$ & $0.0233(7)$ & $-0.0001(6)$ & $0.0062(6)$ & $0.0003(6)$ \\
O9 & $0.0131(8)$ & $0.0363(11)$ & $0.0295(8)$ & $0.0006(7)$ & $0.0032(6)$ & $0.0018(7)$ \\
C2 & $0.0136(9)$ & $0.0281(12)$ & $0.0198(9)$ & $0.0000(8)$ & $0.0031(7)$ & $0.0003(8)$ \\
C3 & $0.0126(9)$ & $0.0326(13)$ & $0.0225(9)$ & $0.0021(9)$ & $0.0028(7)$ & $-0.0008(9)$
\end{tabular}




\begin{tabular}{|c|c|c|c|c|c|c|}
\hline $\mathrm{C} 4$ & $0.0167(10)$ & $0.0247(11)$ & $0.0200(9)$ & $0.0009(8)$ & $0.0011(7)$ & $0.0024(8)$ \\
\hline $\mathrm{C} 5$ & $0.0182(11)$ & $0.0191(11)$ & $0.0178(9)$ & $0.0010(8)$ & $-0.0002(8)$ & $0.0002(8)$ \\
\hline C6 & $0.0183(11)$ & $0.0224(12)$ & $0.0176(9)$ & $0.0003(8)$ & $0.0030(8)$ & $0.0001(8)$ \\
\hline $\mathrm{C} 7$ & $0.0158(11)$ & 0.0167 (11) & $0.0199(9)$ & $0.0000(8)$ & $0.0041(8)$ & $-0.0001(8)$ \\
\hline C8 & $0.0139(10)$ & 0.0177 (11) & $0.0172(8)$ & $0.0003(8)$ & $0.0017(7)$ & $-0.0006(7)$ \\
\hline C9 & $0.0165(11)$ & $0.0222(11)$ & $0.0221(10)$ & $0.0001(8)$ & $0.0013(8)$ & $-0.0009(8)$ \\
\hline $\mathrm{C} 10$ & $0.0227(12)$ & $0.0425(16)$ & $0.0194(10)$ & $-0.0009(10)$ & $0.0045(9)$ & $0.0010(10)$ \\
\hline C4B & $0.0157(10)$ & $0.0203(11)$ & $0.0168(9)$ & $0.0003(8)$ & $-0.0005(7)$ & $-0.0004(8)$ \\
\hline $\mathrm{C} 8 \mathrm{~B}$ & $0.0142(10)$ & $0.0170(11)$ & $0.0184(9)$ & $-0.0003(8)$ & $0.0014(8)$ & $-0.0008(8)$ \\
\hline $\mathrm{C} 1 \mathrm{P}$ & $0.0128(10)$ & $0.0411(15)$ & $0.0195(9)$ & $0.0030(9)$ & $0.0044(7)$ & $0.0003(9)$ \\
\hline $\mathrm{C} 2 \mathrm{P}$ & $0.0278(13)$ & $0.0468(17)$ & $0.0355(12)$ & $-0.0015(12)$ & $0.0054(10)$ & $-0.0102(12)$ \\
\hline C3P & $0.0294(14)$ & $0.075(2)$ & 0.0339 (13) & $0.0016(15)$ & $-0.0012(10)$ & $-0.0245(15)$ \\
\hline $\mathrm{C} 4 \mathrm{P}$ & $0.0223(13)$ & $0.090(3)$ & $0.0218(10)$ & $0.0010(15)$ & $0.0026(9)$ & $0.0013(15)$ \\
\hline C5P & $0.0309(14)$ & $0.069(2)$ & $0.0297(12)$ & $0.0013(14)$ & $0.0033(10)$ & $0.0158(14)$ \\
\hline C6P & $0.0259(13)$ & $0.0431(16)$ & $0.0286(11)$ & $0.0053(11)$ & $0.0042(9)$ & $0.0083(11)$ \\
\hline O1A & $0.0137(7)$ & $0.0304(9)$ & $0.0165(6)$ & $0.0004(6)$ & $0.0017(5)$ & $0.0003(6)$ \\
\hline $\mathrm{O} 4 \mathrm{~A}$ & $0.0154(8)$ & $0.0450(12)$ & $0.0261(8)$ & $0.0013(7)$ & $-0.0028(6)$ & $0.0000(8)$ \\
\hline O5A & $0.0226(9)$ & $0.0426(11)$ & $0.0183(7)$ & $0.0018(8)$ & $-0.0019(6)$ & $-0.0020(7)$ \\
\hline O7A & $0.0159(8)$ & $0.0285(10)$ & $0.0264(8)$ & $-0.0009(6)$ & $0.0071(6)$ & $-0.0023(7)$ \\
\hline O9A & $0.0136(8)$ & $0.0353(11)$ & $0.0310(8)$ & $0.0012(7)$ & $0.0015(6)$ & $-0.0002(7)$ \\
\hline $\mathrm{C} 2 \mathrm{~A}$ & $0.0155(10)$ & $0.0272(12)$ & $0.0195(9)$ & $0.0034(8)$ & $0.0035(8)$ & $0.0009(8)$ \\
\hline $\mathrm{C} 3 \mathrm{~A}$ & $0.0136(9)$ & $0.0376(14)$ & $0.0218(9)$ & $-0.0006(9)$ & $0.0022(7)$ & $0.0000(9)$ \\
\hline $\mathrm{C} 4 \mathrm{~A}$ & $0.0156(10)$ & $0.0258(12)$ & $0.0215(9)$ & $0.0022(9)$ & $-0.0014(7)$ & $-0.0023(8)$ \\
\hline $\mathrm{C} 5 \mathrm{~A}$ & $0.0208(11)$ & $0.0220(12)$ & $0.0174(9)$ & $0.0010(8)$ & $-0.0008(8)$ & $-0.0022(8)$ \\
\hline C6A & $0.0231(12)$ & $0.0217(12)$ & $0.0192(9)$ & $0.0004(9)$ & $0.0045(8)$ & $-0.0015(8)$ \\
\hline C7A & $0.0150(11)$ & $0.0179(11)$ & $0.0240(10)$ & $-0.0005(8)$ & $0.0050(8)$ & $-0.0022(9)$ \\
\hline C8A & $0.0153(11)$ & $0.0174(11)$ & $0.0193(9)$ & $0.0002(8)$ & $0.0015(8)$ & $-0.0009(7)$ \\
\hline C9A & $0.0141(10)$ & $0.0230(12)$ & $0.0238(10)$ & $-0.0005(8)$ & $0.0003(8)$ & $-0.0011(8)$ \\
\hline C10A & $0.0266(13)$ & $0.0455(16)$ & $0.0207(10)$ & $0.0016(11)$ & $0.0069(9)$ & $-0.0020(10)$ \\
\hline C4AA & $0.0154(10)$ & $0.0230(11)$ & $0.0177(9)$ & $0.0015(8)$ & $-0.0001(8)$ & $-0.0026(8)$ \\
\hline C8AA & $0.0151(10)$ & $0.0183(11)$ & $0.0168(9)$ & $0.0001(8)$ & $0.0015(7)$ & $-0.0018(8)$ \\
\hline C1PA & $0.0156(10)$ & $0.0330(13)$ & $0.0185(9)$ & $0.0028(9)$ & $0.0030(8)$ & $-0.0028(9)$ \\
\hline $\mathrm{C} 2 \mathrm{PA}$ & $0.0306(13)$ & $0.0321(13)$ & $0.0210(10)$ & $0.0037(10)$ & $0.0030(9)$ & $-0.0037(9)$ \\
\hline C3PA & $0.0332(14)$ & $0.0327(14)$ & $0.0290(11)$ & $0.0004(11)$ & $0.0030(10)$ & $0.0029(10)$ \\
\hline C4PA & $0.0230(12)$ & $0.0446(16)$ & $0.0233(10)$ & $0.0030(11)$ & $0.0045(9)$ & $0.0020(10)$ \\
\hline C5PA & $0.0284(13)$ & $0.0407(16)$ & 0.0259 (11) & $0.0015(11)$ & $0.0018(9)$ & $-0.0113(11)$ \\
\hline С6РA & $0.0262(12)$ & $0.0306(13)$ & $0.0278(10)$ & $-0.0031(10)$ & $0.0044(9)$ & $-0.0041(10)$ \\
\hline
\end{tabular}

Geometric parameters $\left(A,{ }^{\circ}\right)$

\begin{tabular}{llll}
\hline $\mathrm{O} 1-\mathrm{C} 2$ & $1.473(2)$ & $\mathrm{O} 1 \mathrm{~A}-\mathrm{C} 2 \mathrm{~A}$ & $1.470(2)$ \\
$\mathrm{O} 1-\mathrm{C} 8 \mathrm{~B}$ & $1.349(2)$ & O1A-C8AA & $1.346(2)$ \\
$\mathrm{O} 4-\mathrm{C} 4$ & $1.241(3)$ & O4A-C4A & $1.243(3)$ \\
$\mathrm{O} 5-\mathrm{C} 5$ & $1.339(3)$ & O5A-C5A & $1.340(3)$ \\
$\mathrm{O} 5-\mathrm{H} 5$ & $0.94(3)$ & O5A-H5A & $0.94(3)$ \\
$\mathrm{O} 7-\mathrm{C} 7$ & $1.355(2)$ & O7A-C7A & $1.351(2)$ \\
$\mathrm{O} 7-\mathrm{H} 7$ & $0.95(3)$ & O7A-H7A & $0.94(3)$ \\
$\mathrm{O} 9-\mathrm{C} 9$ & $1.245(3)$ & O9A-C9A & $1.238(2)$
\end{tabular}




\begin{tabular}{|c|c|c|c|}
\hline $\mathrm{C} 2-\mathrm{H} 2$ & 1.0000 & $\mathrm{C} 2 \mathrm{~A}-\mathrm{H} 2 \mathrm{~A}$ & 1.0000 \\
\hline $\mathrm{C} 2-\mathrm{C} 3$ & $1.526(3)$ & $\mathrm{C} 2 \mathrm{~A}-\mathrm{C} 3 \mathrm{~A}$ & $1.520(3)$ \\
\hline $\mathrm{C} 2-\mathrm{C} 1 \mathrm{P}$ & $1.503(3)$ & $\mathrm{C} 2 \mathrm{~A}-\mathrm{C} 1 \mathrm{PA}$ & $1.512(3)$ \\
\hline $\mathrm{C} 3-\mathrm{H} 3 \mathrm{~A}$ & 0.9900 & $\mathrm{C} 3 \mathrm{~A}-\mathrm{H} 3 \mathrm{AA}$ & 0.9900 \\
\hline $\mathrm{C} 3-\mathrm{H} 3 \mathrm{~B}$ & 0.9900 & $\mathrm{C} 3 \mathrm{~A}-\mathrm{H} 3 \mathrm{AB}$ & 0.9900 \\
\hline $\mathrm{C} 3-\mathrm{C} 4$ & $1.504(3)$ & $\mathrm{C} 3 \mathrm{~A}-\mathrm{C} 4 \mathrm{~A}$ & $1.496(3)$ \\
\hline $\mathrm{C} 4-\mathrm{C} 4 \mathrm{~B}$ & $1.462(3)$ & $\mathrm{C} 4 \mathrm{~A}-\mathrm{C} 4 \mathrm{AA}$ & $1.456(3)$ \\
\hline $\mathrm{C} 5-\mathrm{C} 6$ & $1.405(3)$ & $\mathrm{C} 5 \mathrm{~A}-\mathrm{C} 6 \mathrm{~A}$ & $1.400(3)$ \\
\hline $\mathrm{C} 5-\mathrm{C} 4 \mathrm{~B}$ & $1.426(3)$ & $\mathrm{C} 5 \mathrm{~A}-\mathrm{C} 4 \mathrm{AA}$ & $1.428(3)$ \\
\hline $\mathrm{C} 6-\mathrm{C} 7$ & $1.399(3)$ & $\mathrm{C} 6 \mathrm{~A}-\mathrm{C} 7 \mathrm{~A}$ & $1.399(3)$ \\
\hline $\mathrm{C} 6-\mathrm{C} 10$ & $1.506(2)$ & $\mathrm{C} 6 \mathrm{~A}-\mathrm{C} 10 \mathrm{~A}$ & $1.511(2)$ \\
\hline $\mathrm{C} 7-\mathrm{C} 8$ & $1.423(2)$ & $\mathrm{C} 7 \mathrm{~A}-\mathrm{C} 8 \mathrm{~A}$ & $1.422(3)$ \\
\hline $\mathrm{C} 8-\mathrm{C} 9$ & $1.441(3)$ & $\mathrm{C} 8 \mathrm{~A}-\mathrm{C} 9 \mathrm{~A}$ & $1.442(3)$ \\
\hline $\mathrm{C} 8-\mathrm{C} 8 \mathrm{~B}$ & $1.414(3)$ & $\mathrm{C} 8 \mathrm{~A}-\mathrm{C} 8 \mathrm{AA}$ & $1.414(3)$ \\
\hline C9-H9 & 0.9500 & C9A-H9A & 0.9500 \\
\hline $\mathrm{C} 10-\mathrm{H} 10 \mathrm{~A}$ & 0.9800 & $\mathrm{C} 10 \mathrm{~A}-\mathrm{H} 10 \mathrm{D}$ & 0.9800 \\
\hline $\mathrm{C} 10-\mathrm{H} 10 \mathrm{~B}$ & 0.9800 & $\mathrm{C} 10 \mathrm{~A}-\mathrm{H} 10 \mathrm{E}$ & 0.9800 \\
\hline $\mathrm{C} 10-\mathrm{H} 10 \mathrm{C}$ & 0.9800 & $\mathrm{C} 10 \mathrm{~A}-\mathrm{H} 10 \mathrm{~F}$ & 0.9800 \\
\hline $\mathrm{C} 4 \mathrm{~B}-\mathrm{C} 8 \mathrm{~B}$ & $1.401(3)$ & $\mathrm{C} 4 \mathrm{AA}-\mathrm{C} 8 \mathrm{AA}$ & $1.407(3)$ \\
\hline $\mathrm{C} 1 \mathrm{P}-\mathrm{C} 2 \mathrm{P}$ & $1.387(4)$ & $\mathrm{C} 1 \mathrm{PA}-\mathrm{C} 2 \mathrm{PA}$ & $1.392(4)$ \\
\hline $\mathrm{C} 1 \mathrm{P}-\mathrm{C} 6 \mathrm{P}$ & $1.404(4)$ & $\mathrm{C} 1 \mathrm{PA}-\mathrm{C} 6 \mathrm{PA}$ & $1.394(3)$ \\
\hline $\mathrm{C} 2 \mathrm{P}-\mathrm{H} 2 \mathrm{P}$ & 0.9500 & $\mathrm{C} 2 \mathrm{PA}-\mathrm{H} 2 \mathrm{PA}$ & 0.9500 \\
\hline $\mathrm{C} 2 \mathrm{P}-\mathrm{C} 3 \mathrm{P}$ & $1.407(3)$ & $\mathrm{C} 2 \mathrm{PA}-\mathrm{C} 3 \mathrm{PA}$ & $1.393(3)$ \\
\hline $\mathrm{C} 3 \mathrm{P}-\mathrm{H} 3 \mathrm{P}$ & 0.9500 & С 3 PA-H3PA & 0.9500 \\
\hline $\mathrm{C} 3 \mathrm{P}-\mathrm{C} 4 \mathrm{P}$ & $1.384(5)$ & $\mathrm{C} 3 \mathrm{PA}-\mathrm{C} 4 \mathrm{PA}$ & $1.390(4)$ \\
\hline $\mathrm{C} 4 \mathrm{P}-\mathrm{H} 4 \mathrm{P}$ & 0.9500 & C4PA-H4PA & 0.9500 \\
\hline $\mathrm{C} 4 \mathrm{P}-\mathrm{C} 5 \mathrm{P}$ & $1.373(5)$ & $\mathrm{C} 4 \mathrm{PA}-\mathrm{C} 5 \mathrm{PA}$ & $1.385(4)$ \\
\hline $\mathrm{C} 5 \mathrm{P}-\mathrm{H} 5 \mathrm{P}$ & 0.9500 & С5PA-H5PA & 0.9500 \\
\hline $\mathrm{C} 5 \mathrm{P}-\mathrm{C} 6 \mathrm{P}$ & $1.380(3)$ & $\mathrm{C} 5 \mathrm{PA}-\mathrm{C} 6 \mathrm{PA}$ & $1.393(3)$ \\
\hline $\mathrm{C} 6 \mathrm{P}-\mathrm{H} 6 \mathrm{P}$ & 0.9500 & С6РА-Н6РA & 0.9500 \\
\hline $\mathrm{C} 8 \mathrm{~B}-\mathrm{O} 1-\mathrm{C} 2$ & $114.82(16)$ & $\mathrm{C} 8 \mathrm{AA}-\mathrm{O} 1 \mathrm{~A}-\mathrm{C} 2 \mathrm{~A}$ & $116.23(16)$ \\
\hline $\mathrm{C} 5-\mathrm{O} 5-\mathrm{H} 5$ & $105.1(18)$ & $\mathrm{C} 5 \mathrm{~A}-\mathrm{O} 5 \mathrm{~A}-\mathrm{H} 5 \mathrm{~A}$ & $105.8(18)$ \\
\hline $\mathrm{C} 7-\mathrm{O} 7-\mathrm{H} 7$ & $99(2)$ & $\mathrm{C} 7 \mathrm{~A}-\mathrm{O} 7 \mathrm{~A}-\mathrm{H} 7 \mathrm{~A}$ & $101(2)$ \\
\hline $\mathrm{O} 1-\mathrm{C} 2-\mathrm{H} 2$ & 108.3 & $\mathrm{O} 1 \mathrm{~A}-\mathrm{C} 2 \mathrm{~A}-\mathrm{H} 2 \mathrm{~A}$ & 108.9 \\
\hline $\mathrm{O} 1-\mathrm{C} 2-\mathrm{C} 3$ & $109.37(18)$ & $\mathrm{O} 1 \mathrm{~A}-\mathrm{C} 2 \mathrm{~A}-\mathrm{C} 3 \mathrm{~A}$ & $110.40(17)$ \\
\hline $\mathrm{O} 1-\mathrm{C} 2-\mathrm{C} 1 \mathrm{P}$ & $107.42(17)$ & $\mathrm{O} 1 \mathrm{~A}-\mathrm{C} 2 \mathrm{~A}-\mathrm{C} 1 \mathrm{PA}$ & $106.34(17)$ \\
\hline $\mathrm{C} 3-\mathrm{C} 2-\mathrm{H} 2$ & 108.3 & $\mathrm{C} 3 \mathrm{~A}-\mathrm{C} 2 \mathrm{~A}-\mathrm{H} 2 \mathrm{~A}$ & 108.9 \\
\hline $\mathrm{C} 1 \mathrm{P}-\mathrm{C} 2-\mathrm{H} 2$ & 108.3 & $\mathrm{C} 1 \mathrm{PA}-\mathrm{C} 2 \mathrm{~A}-\mathrm{H} 2 \mathrm{~A}$ & 108.9 \\
\hline $\mathrm{C} 1 \mathrm{P}-\mathrm{C} 2-\mathrm{C} 3$ & $115.06(18)$ & $\mathrm{C} 1 \mathrm{PA}-\mathrm{C} 2 \mathrm{~A}-\mathrm{C} 3 \mathrm{~A}$ & $113.22(18)$ \\
\hline $\mathrm{C} 2-\mathrm{C} 3-\mathrm{H} 3 \mathrm{~A}$ & 109.9 & $\mathrm{C} 2 \mathrm{~A}-\mathrm{C} 3 \mathrm{~A}-\mathrm{H} 3 \mathrm{AA}$ & 109.4 \\
\hline $\mathrm{C} 2-\mathrm{C} 3-\mathrm{H} 3 \mathrm{~B}$ & 109.9 & $\mathrm{C} 2 \mathrm{~A}-\mathrm{C} 3 \mathrm{~A}-\mathrm{H} 3 \mathrm{AB}$ & 109.4 \\
\hline $\mathrm{H} 3 \mathrm{~A}-\mathrm{C} 3-\mathrm{H} 3 \mathrm{~B}$ & 108.3 & $\mathrm{H} 3 \mathrm{AA}-\mathrm{C} 3 \mathrm{~A}-\mathrm{H} 3 \mathrm{AB}$ & 108.0 \\
\hline $\mathrm{C} 4-\mathrm{C} 3-\mathrm{C} 2$ & $109.05(17)$ & $\mathrm{C} 4 \mathrm{~A}-\mathrm{C} 3 \mathrm{~A}-\mathrm{C} 2 \mathrm{~A}$ & $111.28(19)$ \\
\hline $\mathrm{C} 4-\mathrm{C} 3-\mathrm{H} 3 \mathrm{~A}$ & 109.9 & $\mathrm{C} 4 \mathrm{~A}-\mathrm{C} 3 \mathrm{~A}-\mathrm{H} 3 \mathrm{AA}$ & 109.4 \\
\hline $\mathrm{C} 4-\mathrm{C} 3-\mathrm{H} 3 \mathrm{~B}$ & 109.9 & $\mathrm{C} 4 \mathrm{~A}-\mathrm{C} 3 \mathrm{~A}-\mathrm{H} 3 \mathrm{AB}$ & 109.4 \\
\hline $\mathrm{O} 4-\mathrm{C} 4-\mathrm{C} 3$ & $121.76(19)$ & $\mathrm{O} 4 \mathrm{~A}-\mathrm{C} 4 \mathrm{~A}-\mathrm{C} 3 \mathrm{~A}$ & $121.2(2)$ \\
\hline $\mathrm{O} 4-\mathrm{C} 4-\mathrm{C} 4 \mathrm{~B}$ & $122.81(18)$ & $\mathrm{O} 4 \mathrm{~A}-\mathrm{C} 4 \mathrm{~A}-\mathrm{C} 4 \mathrm{AA}$ & $122.38(19)$ \\
\hline
\end{tabular}




$\begin{array}{ll}\text { C4B-C4-C3 } & 115.38(19) \\ \text { O5-C5-C6 } & 118.33(17) \\ \text { O5-C5-C4B } & 119.06(18) \\ \text { C6-C5-C4B } & 122.6(2) \\ \text { C5-C6-C10 } & 121.7(2) \\ \text { C7-C6-C5 } & 116.99(17) \\ \text { C7-C6-C10 } & 121.26(19) \\ \text { O7-C7-C6 } & 117.62(17) \\ \text { O7-C7-C8 } & 119.6(2) \\ \text { C6-C7-C8 } & 122.82(18) \\ \text { C7-C8-C9 } & 121.00(18) \\ \text { C8B-C8-C7 } & 118.1(2) \\ \text { C8B-C8-C9 } & 120.86(17) \\ \text { O9-C9-C8 } & 123.58(18) \\ \text { O9-C9-H9 } & 118.2 \\ \text { C8-C9-H9 } & 118.2 \\ \text { C6-C10-H10A } & 109.5 \\ \text { C6-C10-H10B } & 109.5 \\ \text { C6-C10-H10C } & 109.5 \\ \text { H10A-C10-H10B } & 109.5 \\ \text { H10A-C10-H10C } & 109.5 \\ \text { H10B-C10-H10C } & 109.5 \\ \text { C5-C4B-C4 } & 120.9(2) \\ \text { C8B-C4B-C4 } & 120.43(17) \\ \text { C8B-C4B-C5 } & 118.34(19) \\ \text { O1-C8B-C8 } & 116.75(19) \\ \text { O1-C8B-C4B } & 122.21(18) \\ \text { C4B-C8B-C8 } & 121.03(18) \\ \text { C2P-C1P-C2 } & 122.0(2) \\ \text { C2P-C1P-C6P } & 119.7(2) \\ \text { C6P-C1P-C2 } & 118.3(2) \\ \text { C1P-C2P-H2P } & 120.4 \\ \text { C1P-C2P-C3P } & 119.2(3) \\ \text { C3P-C2P-H2P } & 120.4 \\ \text { C2P-C3P-H3P } & 119.9 \\ \text { C4P-C3P-C2P } & 12.9 \\ \text { C4P-C3P-H3P } & 119.8 \\ \text { C3P-C4P-H4P } & 119.8 \\ \text { C5P-C4P-C3P } & 120.4(2) \\ \text { C5P-C4P-H4P } & 119.8 \\ \text { C4P-C5P-H5P } & \\ \text { C4P-C5P-C6P } & \\ \text { C6P-C5P-H5P } & \\ \text { C1P-C6P-H6P } & \\ \text { C5P-C6P-C1P } & \\ \text { C5P-C6P-H6P } & \\ \text { O1-C2-C3-C4 } & \\ & \end{array}$

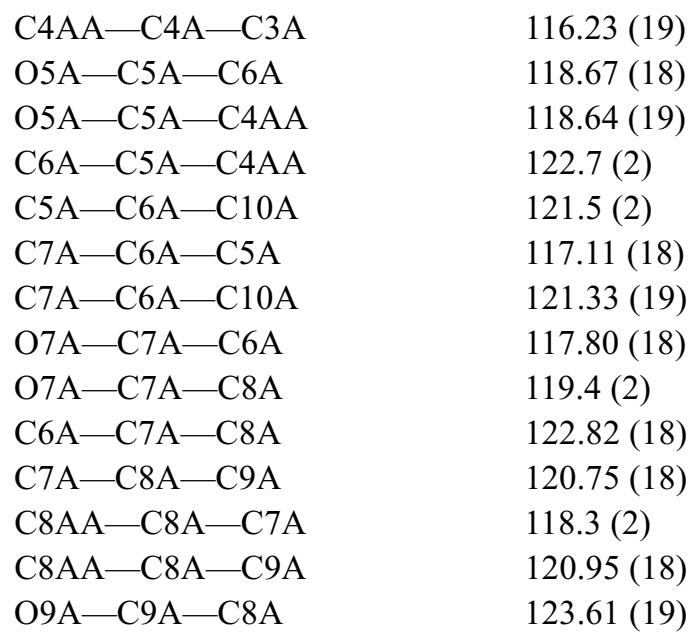

$116.23(19)$

$118.67(18)$

118.64 (19)

122.7 (2)

$121.5(2)$

$117.11(18)$

$121.33(19)$

$117.80(18)$

$119.4(2)$

$122.82(18)$

$120.75(18)$

$118.3(2)$

$120.95(18)$

$123.61(19)$

118.2

118.2

109.5

109.5

109.5

109.5

109.5

109.5

121.3 (2)

$119.93(18)$

$118.26(19)$

116.55 (19)

122.63 (18)

120.81 (18)

121.8 (2)

$119.2(2)$

119.0 (2)

119.8

120.5 (2)

119.8

120.2

119.6 (3)

120.2

119.7

120.6 (2)

119.7

120.3

119.4 (2)

120.3

119.7

120.6 (2)

119.7

$-53.9(3)$ 


\begin{tabular}{|c|c|}
\hline $\mathrm{O} 1-\mathrm{C} 2-\mathrm{C} 1 \mathrm{P}-\mathrm{C} 2 \mathrm{P}$ & $-62.0(3)$ \\
\hline $\mathrm{O} 1-\mathrm{C} 2-\mathrm{C} 1 \mathrm{P}-\mathrm{C} 6 \mathrm{P}$ & $118.7(2)$ \\
\hline $\mathrm{O} 4-\mathrm{C} 4-\mathrm{C} 4 \mathrm{~B}-\mathrm{C} 5$ & $-4.7(4)$ \\
\hline $\mathrm{O} 4-\mathrm{C} 4-\mathrm{C} 4 \mathrm{~B}-\mathrm{C} 8 \mathrm{~B}$ & $-178.3(2)$ \\
\hline $\mathrm{O} 5-\mathrm{C} 5-\mathrm{C} 6-\mathrm{C} 7$ & $-177.4(2)$ \\
\hline $\mathrm{O} 5-\mathrm{C} 5-\mathrm{C} 6-\mathrm{C} 10$ & $0.9(4)$ \\
\hline $\mathrm{O} 5-\mathrm{C} 5-\mathrm{C} 4 \mathrm{~B}-\mathrm{C} 4$ & $6.4(3)$ \\
\hline $\mathrm{O} 5-\mathrm{C} 5-\mathrm{C} 4 \mathrm{~B}-\mathrm{C} 8 \mathrm{~B}$ & $-179.9(2)$ \\
\hline $\mathrm{O} 7-\mathrm{C} 7-\mathrm{C} 8-\mathrm{C} 9$ & $2.1(3)$ \\
\hline $\mathrm{O} 7-\mathrm{C} 7-\mathrm{C} 8-\mathrm{C} 8 \mathrm{~B}$ & $-179.0(2)$ \\
\hline $\mathrm{C} 2-\mathrm{O} 1-\mathrm{C} 8 \mathrm{~B}-\mathrm{C} 8$ & $161.36(19)$ \\
\hline $\mathrm{C} 2-\mathrm{O} 1-\mathrm{C} 8 \mathrm{~B}-\mathrm{C} 4 \mathrm{~B}$ & $-19.5(3)$ \\
\hline $\mathrm{C} 2-\mathrm{C} 3-\mathrm{C} 4-\mathrm{O} 4$ & $-148.6(2)$ \\
\hline $\mathrm{C} 2-\mathrm{C} 3-\mathrm{C} 4-\mathrm{C} 4 \mathrm{~B}$ & $33.8(3)$ \\
\hline $\mathrm{C} 2-\mathrm{C} 1 \mathrm{P}-\mathrm{C} 2 \mathrm{P}-\mathrm{C} 3 \mathrm{P}$ & $-179.6(2)$ \\
\hline $\mathrm{C} 2-\mathrm{C} 1 \mathrm{P}-\mathrm{C} 6 \mathrm{P}-\mathrm{C} 5 \mathrm{P}$ & $179.3(2)$ \\
\hline $\mathrm{C} 3-\mathrm{C} 2-\mathrm{C} 1 \mathrm{P}-\mathrm{C} 2 \mathrm{P}$ & $60.1(3)$ \\
\hline $\mathrm{C} 3-\mathrm{C} 2-\mathrm{C} 1 \mathrm{P}-\mathrm{C} 6 \mathrm{P}$ & $-119.3(2)$ \\
\hline $\mathrm{C} 3-\mathrm{C} 4-\mathrm{C} 4 \mathrm{~B}-\mathrm{C} 5$ & $172.9(2)$ \\
\hline $\mathrm{C} 3-\mathrm{C} 4-\mathrm{C} 4 \mathrm{~B}-\mathrm{C} 8 \mathrm{~B}$ & $-0.7(3)$ \\
\hline $\mathrm{C} 4-\mathrm{C} 4 \mathrm{~B}-\mathrm{C} 8 \mathrm{~B}-\mathrm{O} 1$ & $-8.2(3)$ \\
\hline $\mathrm{C} 4-\mathrm{C} 4 \mathrm{~B}-\mathrm{C} 8 \mathrm{~B}-\mathrm{C} 8$ & $170.9(2)$ \\
\hline $\mathrm{C} 5-\mathrm{C} 6-\mathrm{C} 7-\mathrm{O} 7$ & $177.5(2)$ \\
\hline $\mathrm{C} 5-\mathrm{C} 6-\mathrm{C} 7-\mathrm{C} 8$ & $-2.6(3)$ \\
\hline $\mathrm{C} 5-\mathrm{C} 4 \mathrm{~B}-\mathrm{C} 8 \mathrm{~B}-\mathrm{O} 1$ & $178.0(2)$ \\
\hline $\mathrm{C} 5-\mathrm{C} 4 \mathrm{~B}-\mathrm{C} 8 \mathrm{~B}-\mathrm{C} 8$ & $-2.9(3)$ \\
\hline $\mathrm{C} 6-\mathrm{C} 5-\mathrm{C} 4 \mathrm{~B}-\mathrm{C} 4$ & $-172.4(2)$ \\
\hline $\mathrm{C} 6-\mathrm{C} 5-\mathrm{C} 4 \mathrm{~B}-\mathrm{C} 8 \mathrm{~B}$ & $1.3(4)$ \\
\hline $\mathrm{C} 6-\mathrm{C} 7-\mathrm{C} 8-\mathrm{C} 9$ & $-177.7(2)$ \\
\hline $\mathrm{C} 6-\mathrm{C} 7-\mathrm{C} 8-\mathrm{C} 8 \mathrm{~B}$ & $1.2(3)$ \\
\hline $\mathrm{C} 7-\mathrm{C} 8-\mathrm{C} 9-\mathrm{O} 9$ & $-1.9(4)$ \\
\hline $\mathrm{C} 7-\mathrm{C} 8-\mathrm{C} 8 \mathrm{~B}-\mathrm{O} 1$ & $-179.16(19)$ \\
\hline $\mathrm{C} 7-\mathrm{C} 8-\mathrm{C} 8 \mathrm{~B}-\mathrm{C} 4 \mathrm{~B}$ & $1.7(3)$ \\
\hline $\mathrm{C} 9-\mathrm{C} 8-\mathrm{C} 8 \mathrm{~B}-\mathrm{O} 1$ & $-0.3(3)$ \\
\hline $\mathrm{C} 9-\mathrm{C} 8-\mathrm{C} 8 \mathrm{~B}-\mathrm{C} 4 \mathrm{~B}$ & $-179.5(2)$ \\
\hline $\mathrm{C} 10-\mathrm{C} 6-\mathrm{C} 7-\mathrm{O} 7$ & $-0.8(3)$ \\
\hline $\mathrm{C} 10-\mathrm{C} 6-\mathrm{C} 7-\mathrm{C} 8$ & $179.0(2)$ \\
\hline $\mathrm{C} 4 \mathrm{~B}-\mathrm{C} 5-\mathrm{C} 6-\mathrm{C} 7$ & $1.3(3)$ \\
\hline $\mathrm{C} 4 \mathrm{~B}-\mathrm{C} 5-\mathrm{C} 6-\mathrm{C} 10$ & $179.7(2)$ \\
\hline $\mathrm{C} 8 \mathrm{~B}-\mathrm{O} 1-\mathrm{C} 2-\mathrm{C} 3$ & $53.6(2)$ \\
\hline $\mathrm{C} 8 \mathrm{~B}-\mathrm{O} 1-\mathrm{C} 2-\mathrm{C} 1 \mathrm{P}$ & $179.10(19)$ \\
\hline $\mathrm{C} 8 \mathrm{~B}-\mathrm{C} 8-\mathrm{C} 9-\mathrm{O} 9$ & $179.3(2)$ \\
\hline $\mathrm{C} 1 \mathrm{P}-\mathrm{C} 2-\mathrm{C} 3-\mathrm{C} 4$ & $179.4(2)$ \\
\hline $\mathrm{C} 1 \mathrm{P}-\mathrm{C} 2 \mathrm{P}-\mathrm{C} 3 \mathrm{P}-\mathrm{C} 4 \mathrm{P}$ & $0.3(4)$ \\
\hline $\mathrm{C} 2 \mathrm{P}-\mathrm{C} 1 \mathrm{P}-\mathrm{C} 6 \mathrm{P}-\mathrm{C} 5 \mathrm{P}$ & $0.0(4)$ \\
\hline $\mathrm{C} 2 \mathrm{P}-\mathrm{C} 3 \mathrm{P}-\mathrm{C} 4 \mathrm{P}-\mathrm{C} 5 \mathrm{P}$ & $-0.1(4)$ \\
\hline $\mathrm{C} 3 \mathrm{P}-\mathrm{C} 4 \mathrm{P}-\mathrm{C} 5 \mathrm{P}-\mathrm{C} 6 \mathrm{P}$ & $-0.2(4)$ \\
\hline $\mathrm{C} 4 \mathrm{P}-\mathrm{C} 5 \mathrm{P}-\mathrm{C} 6 \mathrm{P}-\mathrm{C} 1 \mathrm{P}$ & $0.3(4)$ \\
\hline
\end{tabular}

$\begin{array}{ll}\text { O1A-C2A-C1PA-C2PA } & -77.6(3) \\ \text { O1A-C2A-C1PA-C6PA } & 102.6(2) \\ \text { O4A-C4A-C4AA-C5A } & -3.9(4) \\ \text { O4A-C4A-C4AA-C8AA } & -175.9(2) \\ \text { O5A-C5A-C6A-C7A } & -179.7(2) \\ \text { O5A-C5A-C6A-C10A } & -0.6(4) \\ \text { O5A-C5A-C4AA-C4A } & 7.9(3) \\ \text { O5A-C5A-C4AA-C8AA } & 180.0(2) \\ \text { O7A-C7A-C8A-C9A } & 0.2(3) \\ \text { O7A-C7A-C8A-C8AA } & -179.6(2) \\ \text { C2A-O1A-C8AA-C8A } & 162.39(19) \\ \text { C2A-O1A-C8AA-C4AA } & -18.7(3) \\ \text { C2A-C3A-C4A-O4A } & -153.9(2) \\ \text { C2A-C3A-C4A-C4AA } & 30.6(3) \\ \text { C2A-C1PA-C2PA-C3PA } & -178.9(2) \\ \text { C2A-C1PA-C6PA-C5PA } & 179.2(2) \\ \text { C3A-C2A-C1PA-C2PA } & 43.9(3) \\ \text { C3A-C2A-C1PA-C6PA } & -136.0(2) \\ \text { C3A-C4A-C4AA-C5A } & 171.6(2) \\ \text { C3A-C4A-C4AA-C8AA } & -0.4(3) \\ \text { C4A-C4AA-C8AA-O1A } & -6.9(3) \\ \text { C4A-C4AA-C8AA-C8A } & 172.0(2) \\ \text { C5A-C6A-C7A-O7A } & -179.9(2) \\ \text { C5A-C6A-C7A-C8A } & -0.3(3) \\ \text { C5A-C4AA-C8AA-O1A } & -179.1(2) \\ \text { C5A-C4AA-C8AA-C8A } & -0.3(3) \\ \text { C6A-C5A-C4AA-C4A } & -171.3(2) \\ \text { C6A-C5A-C4AA-C8AA } & 0.8(4) \\ \text { C6A-C7A-C8A-C9A } & -179.3(2) \\ \text { C6A-C7A-C8A-C8AA } & 0.8(3) \\ \text { C7A-C8A-C9A-O9A } & -0.8(4) \\ \text { C7A-C8A-C8AA-O1A } & 178.5(2) \\ \text { C7A-C8A-C8AA-C4AA } & -0.5(3) \\ \text { C9A-C8A-C8AA-O1A } & -1.4(3) \\ \text { C9A-C8A-C8AA-C4AA } & 179.7(2) \\ \text { C10A-C6A-C7A-O7A } & 1.1(3) \\ \text { C10A-C6A-C7A-C8A } & -179.4(2) \\ \text { C4AA-C5A-C6A-C7A } & -0.5(4) \\ \text { C4AA-C5A-C6A-C10A } & 178.6(2) \\ \text { C8AA-O1A-C2A-C3A } & 49.0(3) \\ \text { C8AA-O1A-C2A-C1PA } & 172.20(19) \\ \text { C8AA-C8A-C9A-O9A } & 179.0(2) \\ \text { C1PA-C2A-C3A-C4A } & -173.03(19) \\ \text { C1PA-C2PA-C3PA-C4PA } & -0.3(4) \\ \text { C2PA-C1PA-C6PA-C5PA } & -0.7(4) \\ \text { C2PA-C3PA-C4PA-C5PA } & -0.7(4) \\ \text { C3PA-C4PA-C5PA-C6PA } & 0.9(4) \\ \text { C4PA-C5PA-C6PA-C1PA } & -0.3(4) \\ & \end{array}$


supporting information

$\mathrm{C} 6 \mathrm{P}-\mathrm{C} 1 \mathrm{P}-\mathrm{C} 2 \mathrm{P}-\mathrm{C} 3 \mathrm{P} \quad-0.3(4) \quad \mathrm{C} 6 \mathrm{PA}-\mathrm{C} 1 \mathrm{PA}-\mathrm{C} 2 \mathrm{PA}-\mathrm{C} 3 \mathrm{PA} \quad 0.9(4)$

Hydrogen-bond geometry $\left(\AA,{ }^{\circ}\right)$

\begin{tabular}{lllll}
\hline$D-\mathrm{H} \cdots A$ & $D-\mathrm{H}$ & $\mathrm{H} \cdots A$ & $D \cdots A$ & $D-\mathrm{H} \cdots A$ \\
\hline $\mathrm{C} 3-\mathrm{H} 3 B \cdots \mathrm{O} 9 A^{\mathrm{i}}$ & 0.99 & 2.32 & $3.252(3)$ & 158 \\
$\mathrm{C} 3 A-\mathrm{H} 3 A B \cdots \mathrm{O} 9^{\mathrm{ii}}$ & 0.99 & 2.31 & $3.248(3)$ & 157 \\
$\mathrm{O} 7 A-\mathrm{H} 7 A \cdots \mathrm{O} 9 A$ & $0.94(3)$ & $1.67(3)$ & $2.572(2)$ & $159(4)$ \\
$\mathrm{O} 5-\mathrm{H} 5 \cdots \mathrm{O} 4$ & $0.94(3)$ & $1.70(3)$ & $2.579(2)$ & $154(3)$ \\
$\mathrm{O} 7-\mathrm{H} 7 \cdots \mathrm{O} 9$ & $0.95(3)$ & $1.67(3)$ & $2.585(2)$ & $162(3)$ \\
$\mathrm{O} 5 A-\mathrm{H} 5 A \cdots \mathrm{O} 4 A$ & $0.94(3)$ & $1.70(3)$ & $2.574(2)$ & $153(3)$
\end{tabular}

Symmetry codes: (i) $-x+3 / 2, y-1 / 2,-z+1 / 2$; (ii) $-x+1 / 2, y+1 / 2,-z+1 / 2$. 